\title{
Density of Electron States and Volume of Solution of Hydrogen in SiC Alloys
}

\author{
Juana L. Gervasoni ${ }^{1}$ and Juan C. Furnari ${ }^{2}$ \\ 1. Centro Atómico Bariloche, CNEA (Comisión Nacional de Energía Atómica), San Carlos de Bariloche 8400, Río Negro, Argentina \\ 2. Centro Atómico Ezeiza, CNEA (Comisión Nacional de Energía Atómica), Buenos Aires, Argentina
}

Received: June 06, 2013/ Accepted: August 12, 2013 / Published: February 28, 2014.

\begin{abstract}
The new reactor concepts are characterized by higher efficiency, better utilization of nuclear fuel and nuclear waste minimization. This approach means that it is necessary to continue a continued research and test of new materials in order to apply them in new reactors. In this study, the authors focused on the analysis of SiC alloys because, due to their particular properties, this alloy can be used in high temperature conditions where the pure silicon, semiconductor material par excellence, is inadequate to support them.
\end{abstract}

Key words: Hydrogen, volume of solution, electronic states.

\section{Introduction}

$\mathrm{SiC}$ (silicon carbide) has a diamond structure, despite the different size of $\mathrm{C}$ and $\mathrm{Si}$ which could prevent it. It is almost as hard as diamond. It is a refractory semiconductor material and has many advantages for use in devices that involve working regime in extreme conditions of temperature, voltage and frequency. This material can withstand an electric field gradient up to eight times higher than silicon or gallium arsenide without rupture. This higher value of breakdown electric field does prove useful in the manufacture of components that operate at high power, and a candidate for study reactor components [1].

$\mathrm{SiC}$ is a compound solid alloy, based on that of the host structure. (C diamond shape) thereof is changed by atoms of silicon atoms, provided that the hole that is left is similar to the size of atom will occupy it. For this reason the model can be used as described below [2].

Due to the high saturation velocity of charge carriers $\left(2.0 \times 107 \mathrm{~cm}^{-1}\right)$, it is possible to use $\mathrm{SiC}$ devices operating at high frequencies, whether radio

Corresponding author: Juana L. Gervasoni, Ph.D., research field: materials science. E-mail: gervason@cab.cnea.gov.ar. frequencies or microwaves. Finally, a hardness of $\sim 9$ on the Mohs scale that provides mechanical strength to electrical properties makes SiC-based devices to offer numerous benefits over other semiconductors [2].

The silicon carbide is present in a variety of polymorphic crystalline forms, designated generally as $\beta$-SiC and the cubic form $\alpha$-SiC for hexagonal and rhombohedral varieties (Fig. 1) [3]. The alpha form is more stable at temperatures above $2,000{ }^{\circ} \mathrm{C}$, whereas the cubic $\beta-\mathrm{SiC}$ is the most common when silicon carbide is produced at lower temperatures. We will focus on our study in the alpha structure.

On the other hand, hydrogen is a very active chemical element that reacts strongly with metallic substances. His behavior as an impurity in metallic systems has characteristics that differentiate it from others, for example its high mobility. This feature allows it to respond to the stress fields generated by chemical potential gradients, migrating to establish an uneven equilibrium concentration, leveling the chemical potential (Gorsky effect). That is, because the presence of hydrogen dramatically alter the properties of the materials and their alloys, it is necessary to carefully analyze the behavior of it in the systems involved. 


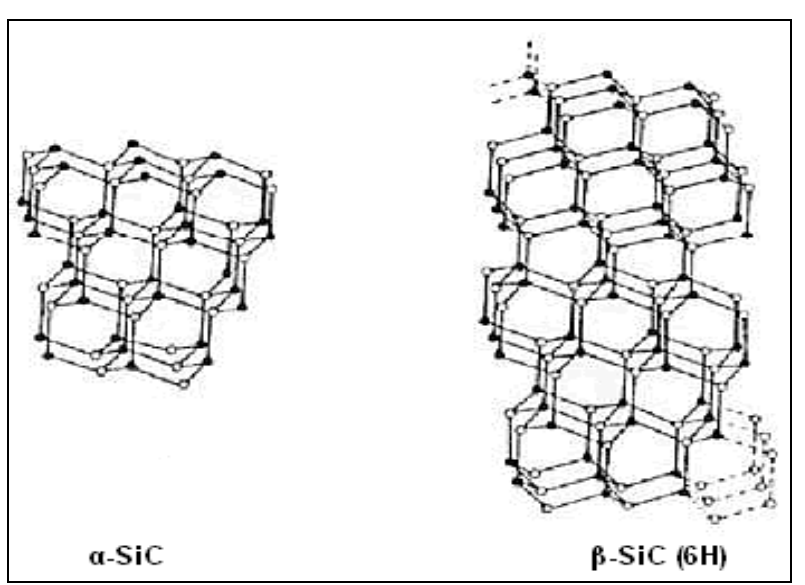

Fig. $1 \mathrm{SiC}$ in their two most common crystal shapes [3].

\section{Modeling}

The screening charge density is a fundamental quantit for it is involved in the microscopic and macroscopic properties of materials. This charge density is also important to understand the relationships between these properties in metal-hydrogen systems. The calculation of this quantity involves important numerical complications, including severe discrepancies observed between the results obtained by different authors, generally resulting from using different pseudo-potential or different calculation techniques.

Alternatively, we proposed a new parameterization, free from the mentioned difficulties, which is characterized by few parameters with clear physical meaning.

The calculation program we use to calculate the screening charge density implements the method of Kohn and Sham [2, 3], in the density functional formalism. The material is modelled by the so-called jellium model [4], in which the metal can be replaced by a uniform electron gas with a background of positive charge of the same average density as the gas, in order to maintain the neutrality of the metal [4]:

$$
n_{o}^{-1}=\frac{4}{3} \pi r_{s}^{3}
$$

(Atomic units are used throughout).

In a previous work [5], we have adopted as $r_{s}$ for each element of the periodic table the one obtained by the bulk modulus B that comes from the (inverse of the) compression $K$ of the electronic density at interstitial positions. The principal contribution to this compression is due to the free electrons, and is written as Ref. [5]:

$$
B=\frac{1}{K}=-V \frac{\partial P}{\partial V}
$$

Taking these values, and using the parameterization obtained in our previous work [5], we have for the induced charge density, or equivalently, for the integrated induced density charge $Q(R)$, the following:

$$
Q(R)=4 \pi \int_{0}^{R} n(r) r^{2} d r
$$

with the appropriate boundary conditions.

\subsection{Volume of Dissolution of Hydrogen}

The volume of dissolution of hydrogen $V_{H}$ is important as responsible for the changes in the mechanical properties of the material since it determines the location of hydrogen dissolved in the material, e.g. interstitial positions, vacancies, or other surface defects). It is defined as [5]:

$$
v_{H}=\frac{V_{H}}{\Omega}=-\frac{1}{B} \sum_{i} \frac{1}{3} \frac{R_{i}}{\Omega} \frac{\partial E}{\partial R_{i}}
$$

where, $\Omega$ is the volume per metal atom, $\underline{B}$ is the bulk modulus and deformation, $\partial R_{i}$ is an arbitrary deformation in the position of metal ions. $\partial E$ is the energy change given by the electrostatic interaction between the ionic core of the metal ion and the impurity placed at the origin:

$$
\partial E=\sum_{i} Z_{i}\left(\int \frac{\Delta n(r)-Z_{o} \partial(r)}{\left|r-R_{i}\right|^{3}}\left(R_{i}-r\right) d^{3} r\right) \partial R_{i}
$$

$Z_{i}$ is the effective charge of the ion located in $R_{\mathrm{i}}$, and $\partial(r)$ is the density of the induced impurity at $Z_{o}$.

\subsection{Variation of the Density of States}

Another value obtained from $\Delta n$ is the variation of the density of states induced by hydrogen in a metal, $\partial g$ $(k)$. The importance of this amount is given due to its relevance on how the electronic structure of the metal 
is affected when hydrogen is added. The Jellium program calculates the induced density of states by the self-consistent method, which result is to characterize the asymptotic behavior of the radial part of the wave function of the impurity [6] as:

$$
R_{k l_{r \rightarrow \infty}}=\operatorname{sen}\left(k r-\frac{1}{2} l \pi+\partial_{l}(k)\right)
$$

The number $l$ is the angular momentum eigenvalue and $k$ is the impulse of the particle.

With the previous phase shifts $\partial_{l}(k)$ and the adequate boundary conditions, the analytical expression for $\Delta g$, valid in the range $0<k<k_{F}$, can be written as the sum of the partial contributions of:

$$
\Delta g_{Z r}\left(\frac{k}{k_{l}} ; l\right)=\Delta g_{o}+A_{s} \operatorname{tgh}\left(\omega \cdot x_{o}\right)+A_{s} \operatorname{tgh}\left[\omega\left(x-x_{o}\right)\right]
$$

where, we have defined $x=k / k_{F}$, with $k_{F}$ is the Fermi wave vector [6].

Note that the induced density of states obtained by our model contribute to the understanding of the electronic structure induced by any impurity in the material, and is related to the excitation spectrum caused by it in the metal [7].

\section{Results}

In a previous study [5], for each element of the periodic table we have evaluated for the radius $r_{s}$ values obtained by the inverse of the compression $K$, calculated from the electron density in interstitial positions. But for these pseudo-binary alloys, another way to assign the value of $r_{s}$ is from the Fermi energy of the system $[8,9]$. According to this criterion, the value of $r_{s}$ for $\mathrm{SiC}$ is 3.472 atomic units.

A check performed to see the above properties in the two crystallographic forms of $\mathrm{SiC}$ was as follows: In a previous work [10], we estimate these quantities regardless of the temperature at which each crystal form is stable. The $\alpha-\mathrm{SiC}$ is stable at high temperatures. We used the Jellium program for this range of temperatures, which is shown in Fig. 2. We observe that the variation with temperature is negligible, meaning that the Jellium model is still applicable. This is because the temperature is one factor of homogenization of the system, which favors the Jellium-type modeling, whose main contribution is due to the free electrons.

Then, using this result, the values of volume (Table 1) and heat (Table 2) of the solution of hydrogen in pure $\mathrm{SiC}$ elements are compared both, with experimental data and with more sophisticated calculations, as tight binding, showing a reasonable agreement, within the model assumptions.

Finally, in Fig. 3 the authors show the induced density of states, previously obtained with zero pressure and temperature, now with non-zero temperature, noting that with this model, the temperature dependence is negligible.

\section{Conclusions}

In this study we focused on the analysis of SiC alloys, since due to their specific properties, this alloy can be used in high temperature conditions where silicon cannot function. In particular, we study factors of microchemistry structure and their evolution in the relationship between structure and composition of these materials, in response to the introduction of impurities of hydrogen in its matrix.

Since it can be analyzed as a solid solution, in this work we managed to model and calculate the electron density, and compared it with that of the pure elements. Depending on the variation of the charge density $(\Delta n)$ and on the characteristic parameters of the effective jellium model, we obtained the solution volume of hydrogen (Table 1) and the solid solution of hydrogen (Table 2) in the alloy, and compared it with those obtained by the semi-empirical band theory model and with the experimental values of their components. They give reasonable values to test with the eventual experimental values of both quantities in the alloy.

In order to compare the density of states between pure $\mathrm{C}$ and pure $\mathrm{Si}$ and the alloy $\mathrm{SiC}$, it is necessary to perform the calculations in identical circumstances, i.e., in an electronic structure corresponding to spherical symmetry. 


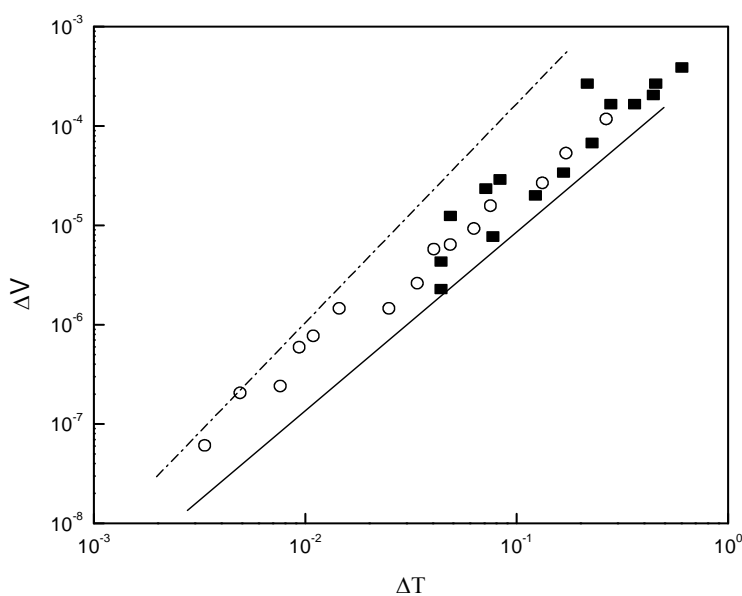

Fig. 2 Change in volume of $\mathrm{H} \mathrm{SiC} \mathrm{solution,} \mathrm{with} \mathrm{respect} \mathrm{to}$ the dimensionless temperature $\Delta T=\left(T-T_{I N F}\right) / T_{0}$, where $T$ is the temperature in the fluid in an arbitrary position $r$ and time $t ; T_{I N F}$ is the temperature infinitely far of impurity, and $T_{0}=L / C_{p}$ is the over-cooling unit, determined from the melting latent heat $\mathrm{L}$ and the specific heat $C_{P}$ fluid.

Table 1 Volume of solution $\mathrm{VH}_{\text {jell }}$ obtained in this work, compared with experimental values $\left(\mathrm{VH}_{\text {exp }}\right)$ and tight binding model $\left(\mathrm{VH}_{t b}\right)$.

\begin{tabular}{llll}
\hline Sample & $V_{\text {Hjell }}\left(\AA^{3}\right)$ & $V_{\text {Hexp }}\left(\AA^{3}\right)$ & $V_{\text {Htb }}\left(\AA^{3}\right)$ \\
\hline $\mathrm{Si}$ & 3.23 & $3.25(6)$ & $2.96(8)$ \\
$\mathrm{C}$ & 2.03 & $2.36(6)$ & $1.17(8)$ \\
$\mathrm{Si}-\mathrm{C}$ & 2.24 & - & $4.46(8)$ \\
\hline
\end{tabular}

Table 2 Solid solution heat value of hydrogen, $\Delta H_{\text {jell, }}$, compared to experimental values $\left(\Delta H_{\text {exp }}\right)$.

\begin{tabular}{lll}
\hline Sample & $H_{\text {jell }}(\mathrm{kJ} / \mathrm{mol})$ & \multicolumn{1}{c}{$H_{\text {exp }}(\mathrm{kJ} / \mathrm{mo})$} \\
\hline $\mathrm{C}$ & -52.00 & $-52.37(7)$ \\
$\mathrm{Si}$ & -54.00 & $-54.08(7)$ \\
$\mathrm{Si}-\mathrm{C}$ & -50.40 & - \\
\hline
\end{tabular}

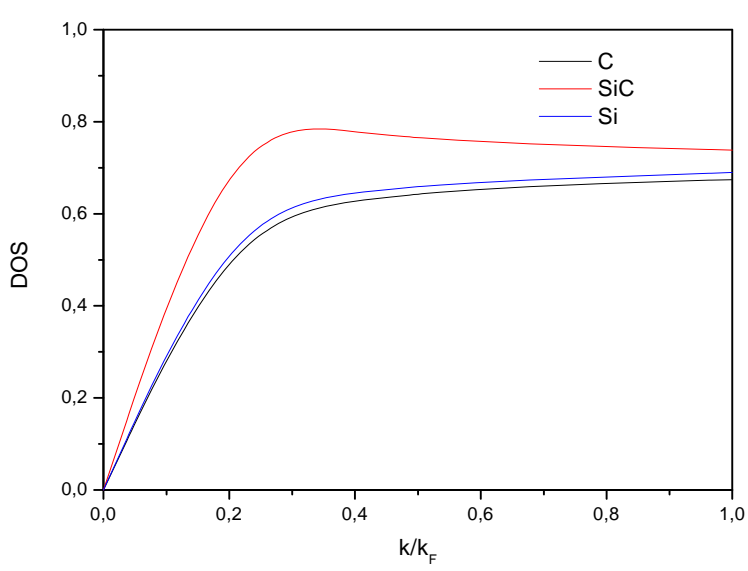

Fig. 3 Induced DOS (density of states) of hydrogen in $\mathrm{C}$, Si and $\mathrm{SiC}$.
The calculations of the variation of the induce density of states due to the presence of hydrogen, show the strong interaction between it and each state of the components as well as the alloy that form the system.

With the Jellium model, we have obtained a simple and useful parameterization of the density induced states, for hydrogen in $\mathrm{SiC}$ matrix, incorporating reasonably well the effects due to the local environment of the impurity. It provides a great simplicity in the analysis, also providing a way to calculate the corrections necessary for this approach.

\section{Acknowledgments}

This work was partially supported by CNEA, CONICET and Univ. Nac. de Cuyo, Argentina.

\section{References}

[1] N. Klyamkin, V.N. Ver, Thermodynamic particularities of some CeNi 5 -based metal hydride systems with high dissociation pressure, in: Proceedings of the International Symposium on Metal-Hydrogen Systems-Fundamentals and Applications, 1995, p. 479.

[2] W. Kohn, L.J. Sham, Self-consistent equations including exchange and correlation effects, Physical Review 140 (4A) (1965) A1133-A1138.

[3] P. Hohenberg, W. Kohn, Inhomogeneus electron gas, Physical Review 136 (3B) (1964) B864-B871.

[4] M.J. Stott, E. Zaremba, Quasiatoms: An approach to atoms in nonuniform electronic systems, Physical Review B 22 (1980) 1564.

[5] S.A. Serebrinsky, J.L. Gervasoni, J.P. Abriata, V.H. Ponce, Characterization of the electronic density of metals in terms of the bulk modulus, Journal of materials Science 33 (1) (1998) 167-171.

[6] S.A. Serebrinsky, J.L. Gervasoni, Density of states induced by a hydrogenic impurity in a metal, International Journal of Hydrogen Energy 29 (5) (2004) 497-500.

[7] M. Okada, T. Kuriiwa, A. Kamegawa, H. Takamura, Role of intermetallics in hydrogen storage materials, Material Science and Engineering A (329-331) (2002) 305-312.

[8] L. Nordstrom, M.S.S. Brooks, B. Johansson, Theoretical study of the enhanced paramagnetism in $\mathrm{CeNi}_{x}(x=1,2$, and 5), Physical Review B 46 (1992) 3458-3464.

[9] R. Griessen, R. Feenstra, Volume changes during hydrogen absorption in metals, Journal of Physics F: Metal Physics 15 (1985) 1013-1019.

[10] L.M. Bianco, J.L. Gervasoni, J.C. Furnari, Study of new materials resistent to radiation, in: Annual Meeting Minutes (Actas Reunión Anual) AATN, Trabajo, 2010. 